\title{
Implicações da Produção do Conhecimento em Psicologia sobre Álcool e Outras Drogas
}

\author{
Carolina dos Reis ${ }^{1}$ \\ ${ }^{1}$ Universidade Federal do Rio Grande do Sul, RS, Brasil.
}

Resumo: Este artigo integra o dossiê comemorativo dos 40 anos da revista Psicologia: Ciência e Profissão e tem por objetivo analisar as publicações da revista sobre a temática de álcool e outras drogas no período de 2005 a 2018. Para tanto, foram identificados 18 artigos dentre todos os publicados nos números regulares da revista que tinham esse tema como foco central. Os artigos foram lidos na íntegra e categorizados de acordo com quatro subtemáticas: a) motivos que levam ao consumo de álcool e outras drogas; b) produção de verdades sobre os usuários de drogas; c) prevenção e tratamento; e d) análise das políticas sobre drogas. Ao final, reflete-se sobre as implicações da produção do conhecimento em Psicologia na construção de políticas sobre drogas.

Palavras-chave: Produção do Conhecimento em Psicologia, Álcool e Drogas, Políticas sobre Drogas, Revista Psicologia: Ciência e Profissão.

\section{Implications of the Production of Psychological Knowledge on Alcohol and Other Drugs}

\begin{abstract}
This paper is part of the dossier that celebrates the 40th anniversary of Psicologia: Ciência e Profissão journal. It aims to analyze reports about alcohol and other drugs published in the journal from 2005 to 2018. In order to do that, 18 papers were identified from all the ones published in regular issues of the journal that had this subject as their central focus. The papers were fully read and categorized in accordance with four sub-themes: a) reasons leading to the consumption of alcohol and other drugs; b) production of truths about drug users; c) prevention and treatment; and d) analysis of drug-related policies. At the end, there are some reflections on the implications of knowledge production in Psychology on the formulation of drug-related policies.
\end{abstract}

Keywords: Knowledge Production in Psychology, Alcohol and Drugs, Drug-Related Policies, Psicologia: Ciência e Profissão Journal. 


\title{
Implicaciones de la Producción de Conocimiento en Psicología sobre el Alcohol y Otras Drogas.
}

\begin{abstract}
Resumen: Este artículo es parte del dossier conmemorativo del $40^{\circ}$ aniversario de la revista Psicologia: Ciência e Profissão y tiene como objetivo analizar las publicaciones de la revista sobre el tema del alcohol y otras drogas en el período comprendido entre 2005 y 2018. Para este propósito, se identificaron 18 artículos entre todos artículos publicados en los números regulares de la revista que se centraron en este tema. Los artículos se leyeron en su totalidad y se clasificaron de acuerdo con cuatro subtemas: a) razones que conducen al consumo de alcohol y otras drogas; b) producción de verdades sobre los consumidores de drogas; c) prevención y tratamiento; y d) análisis de políticas de drogas. Al final, reflexiona sobre las implicaciones de la producción de conocimiento en Psicología en la construcción de políticas de drogas.
\end{abstract}

Palabras clave: Producción de Conocimiento en Psicología, Alcohol y Drogas, Política de Drogas, Revista Psicologia: Ciência e Profissão.

Este artigo integra o dossiê alusivo aos 40 anos da revista Psicologia: Ciência e Profissão, editorada pelo Conselho Federal de Psicologia, com foco na atuação profissional das(es/os) psicólogas(es/os) ${ }^{1}$ e o compartilhamento e impulsionamento da produção de saberes nas mais diversas áreas da Psicologia. O dossiê teve por objetivo analisar os deslocamentos na produção de conhecimentos e práticas em Psicologia que marcam modificações político-sociais. Para tanto, tomou-se como material de pesquisa as publicações realizadas entre 2005 e 2018 dos números regulares da revista. Essa primeira análise foi realizada por Guareschi, Galeano e Bicalho (2020) a partir da leitura dos resumos e palavras-chave de 866 artigos que integraram os 56 números da revista publicados no período de abrangência da pesquisa. Esses artigos foram categorizados em 19 temáticas que emergiram das questões centrais abordadas pelos trabalhos.

$\mathrm{O}$ presente artigo tem por objetivo analisar as publicações relacionadas especificamente à temática de álcool e outras drogas. No período de 2005 a 2018, foram publicados 18 artigos tendo esse tema como foco. Embora esse não seja um grande volume de publicações, a análise dessa temática deve-se às recentes transformações na chamada "Lei Antidrogas", a Lei no 11.343, de 23 de agosto de 2006, sancionadas pela Lei no 13.840 , em 5 de junho de 2019. Dentre as principais mudanças, está o reconhecimento das comunidades terapêuticas como aparelhos do Sistema Nacional de Políticas sobre Drogas, voltadas para a oferta de internação "voluntária", na perspectiva da abstinência. Ademais, o próprio texto da Lei Antidrogas inclui a previsão de internação involuntária como recurso terapêutico para tratamento de "usuários ou dependentes" de drogas.

Além dessas recentes transformações, a política sobre drogas e as formas de atenção a pessoas que fazem uso de substâncias psicoativas têm estado constantemente em pauta dentro do Sistema Conselhos de Psicologia, focalizando-se a análise dos modelos de cuidado ofertados a pessoas com sofrimentos decorrentes do uso de álcool e outras drogas; a intenção é refletir sobre as formas de exclusão e os tratamentos cruéis, desumanos e degradantes que têm sido produzidos em nome da proteção e do cuidado. O Conselho Federal de Psicologia tem afirmado a necessidade de mudança na lógica proibicionista que vem orientando a construção de políticas sobre drogas no Brasil (CFP, 2012; 2014; 2018; CFP \& Crepop, 2019) e de um maior investimento em práticas de redução de danos como tecnologia prioritária na atenção a pessoas que fazem uso de drogas (CFP, 2010; 2012; 2014; 2018). Ademais, tem se posicionado em defesa do cuidado em liberdade, denunciando práticas violadoras de direitos presentes dentro de comunidades terapêuticas em todo o país (CFP, 2011; CFP, MNPCT, \& MPF, 2018).

A exemplo disso, no período de abrangência desta pesquisa, foram realizadas duas inspeções nacionais

${ }^{1}$ O Sistema Conselhos de Psicologia vem há alguns anos gendrando a escrita de seus textos no feminino por reconhecer que a maioria absoluta da profissão é composta por mulheres. 
em comunidades terapêuticas: a primeira em 2011 e a segunda em 2017. Em ambos os relatórios das inspeções constatam-se violações de direitos. O relatório mais recente é composto por denúncias de práticas de castigo e punição a internos, no formato de isolamento físico ou confinamento, violências físicas, práticas de trabalho forçado e em condições degradantes. Há, ainda, denúncias de violação da liberdade religiosa e da diversidade sexual, além de internações involuntárias sem laudos médicos, requeridos para essa modalidade de internação. Segundo o documento, algumas comunidades terapêuticas realizam internamento forçado por meio de uma equipe que vai à residência da pessoa e a imobiliza, fazendo uso tanto de violência física quanto de contenção por meio da aplicação de medicamentos, prática que pode configurar crime de sequestro e cárcere privado qualificado, conforme o artigo 148 do Código Penal (CFP, MNPCT, \& MPF, 2018). Mesmo nas comunidades que anunciam realizar somente internações voluntárias, o relatório mostra que não existem condições reais de os internos interromperem o "tratamento", pois há imposições de barreiras, tais como: retenção de documentos, intervenções para dissuadir a vontade apresentada, não viabilização de transporte para a saída de instituições isoladas dos perímetros urbanos e até ausência de política ativa de informação e transparência que permita à pessoa internada realizar uma tomada de decisão autônoma e soberana sobre quando cessar o "tratamento" 2 (CFP, MNPCT, \& MPF, 2018).

Por fim, denuncia-se a prática de internação de adolescentes, em contrariedade ao estabelecido pelo Ministério da Saúde por meio da Portaria $\mathrm{n}^{\circ}$ $3.088 / 2011$, que determina que comunidades terapêuticas só podem acolher adultos (inciso II do artigo 9o). Essa determinação do Ministério da Saúde sustenta-se no reconhecimento de que as comunidades terapêuticas são equipamentos que não possuem condições de oferecer proteção integral a crianças e adolescentes, uma vez que não atendem às obrigações mínimas estabelecidas no artigo 94 do Estatuto da Criança e do Adolescente (Lei no 8.069/1990). Para o Conselho Federal de Psicologia, esse cenário torna ainda mais temerário o aumento das formas de financiamento de comunidades terapêuticas por meio de recursos públicos, principalmente quando acompanhado pelo sucateamento dos Centros de Atenção Psicossocial - Álcool e Drogas e pelo esfacelamento da Rede de Atenção Psicossocial (CFP, 2018).

Frente a isso, reforça-se a importância de tomar como objeto de análise a produção de conhecimento em Psicologia vinculada à temática de álcool e outras drogas; em especial, consideram-se aqui as publicações da revista Psicologia: Ciência e Profissão, implicada na construção e legitimação de modelos de cuidado e de formas de gestão e controle do crime. Para desenvolver esta análise, partiu-se primeiro de um movimento de desnaturalização da relação entre drogas e produção do conhecimento em Psicologia, para deixar de tomar tal relação como evidência e passar a colocá-la como um problema a ser pensado. Trata-se, portanto, de considerar que as drogas, sejam suas formas de usos, as práticas terapêuticas a elas vinculadas ou mesmo a construção de políticas de segurança pública, não são "objetos naturais" de estudo ou de intervenção da Psicologia. Essa aproximação foi sendo construída historicamente e está inserida em jogos de poder e de interesse atravessados por questões políticas, econômicas e culturais que compõem o nosso presente. Por isso, colocar em análise essa relação significa tomar nossas próprias práticas como uma problemática e investir no necessário exercício de reflexão ético-política sobre os efeitos dessas produções.

\section{Procedimento de análise}

Para a realização desta análise, tomamos como base o levantamento previamente realizado por Guareschi, Galeano e Bicalho (2020), que identificaram 18 artigos vinculados ao tema. Realizamos a leitura completa de todos os artigos e seu tabelamento segundo: data e número da publicação, estado de origem ${ }^{3}$, palavras-chave, objetivos, metodologia, referenciais teóricos, temática central e principais resultados apresentados. Após essa organização, os artigos foram separados em subcategorias, considerando-se suas temáticas centrais.

No que se refere à distribuição territorial, dentre os artigos analisados, quatro são oriundos de estados

\footnotetext{
${ }^{2}$ Frente a isso, a pessoa perde o poder de decisão de encerrar o tratamento, assegurado pela Lei no 10.216/2001, art.7o (CFP, 2017).

${ }^{3}$ Dois artigos tinham autores oriundos de diferentes estados do país. Nesses casos, os artigos foram classificados segundo o local de origem da maior parte dos autores. Em um deles, no entanto, havia a mesma quantidade de autores de diferentes estados, e o artigo foi classificado conforme o estado de origem do primeiro autor.
} 
do Sul do país, sendo três do Rio Grande do Sul, dois de Santa Catarina e um do Paraná; seis são da região Sudeste, sendo dois de Minas Gerais, dois de São Paulo, um do Rio de Janeiro e um do Espírito Santo; por fim, os outros seis artigos provêm de estados do Nordeste, sendo três da Paraíba, um do Piauí, um de Pernambuco e um do Ceará. A Tabela 1 indica a distribuição dos artigos ao longo dos anos, números da revista em que foram publicados e as palavras-chave de cada um deles.

Quanto aos referenciais teóricos, há forte presença de estudos sustentados em pesquisas experimentais, em especial no que se refere a análises de caráter comportamental e atitudinal frente às drogas. Outra presença marcante é a de artigos fundamentados na Teoria das Representações Sociais, seja para análise das percepções dos usuários sobre si, seja para análise da percepção de trabalhadores das políticas de saúde. Em menor proporção, temos a presença de aportes teóricos psicanalíticos e de pesquisas sustentadas no campo da redução de danos. Em todos os artigos, há referência a legislações diversas relacionadas a políticas sobre drogas no campo da saúde, da assistência social e da segurança pública.

Já no que se refere às subcategorias, os artigos foram divididos em quatro temas: a) motivos que levam ao consumo de álcool e outras drogas; b) produção de verdades sobre os usuários de drogas; c) prevenção e tratamento; e d) análise das políticas sobre drogas. Importante ressaltar que vários dos artigos dialogam com mais de uma categoria, tendo sido agrupados de acordo com a temática que assumia maior volume nas análises realizadas. Apresenta-se a seguir o detalhamento das discussões presentes em cada uma das quatro temáticas.

\section{Tabela 1.}

Palavras-chave dos artigos.

\begin{tabular}{|c|c|c|c|}
\hline & Ano & Número & Palavras-chave \\
\hline 1 & 2005 & 1 & alcoolismo, religiosidade, psicologia analítica, estudo de caso \\
\hline 2 & 2006 & 4 & droga, ilegalismo, disciplina, norma \\
\hline 3 & 2007 & 1 & consumismo, criminalidade, psicanálise, transdisciplinaridade \\
\hline 4 & 2012 & 1 & representação social, alcoolismo, índios, profissionais de saúde, drogas \\
\hline 5 & 2012 & 1 & psicologia social, meios de comunicação, drogas, cocaína, crack \\
\hline 6 & 2013 & 2 & $\begin{array}{l}\text { tabagismo, psicologia organizacional, terapia cognitivo-comportamental, saúde } \\
\text { mental }\end{array}$ \\
\hline 7 & 2013 & 3 & $\begin{array}{l}\text { prevenção do abuso da droga, reabilitação da droga, formulação de políticas, } \\
\text { legislação em saúde mental }\end{array}$ \\
\hline 8 & 2014 & 2 & drogas, concepção, profissionais da saúde, política de saúde, intervenção precoce \\
\hline 9 & 2014 & 3 & álcool etílico, trânsito (transgressões), atitudes, prevenção \\
\hline 10 & 2015 & 1 & uso de drogas, ambiente de trabalho, intervenções, trabalhadores \\
\hline 11 & 2015 & 3 & $\begin{array}{l}\text { comunidade terapêutica, transtornos relacionados ao uso de substâncias, } \\
\text { cooperação do paciente }\end{array}$ \\
\hline 12 & 2015 & 3 & bebidas alcoólicas, comportamento, psicologia social \\
\hline 13 & 2016 & 1 & drogas, usuário de drogas, dependência química, representações sociais \\
\hline 14 & 2016 & 2 & $\begin{array}{l}\text { bebida alcoólica, direção automotiva, blitz, educação para o trânsito, avaliação } \\
\text { psicológica de motorista, Programa Madrugada-Viva }\end{array}$ \\
\hline 15 & 2017 & 2 & $\begin{array}{l}\text { atenção primária à saúde, saúde mental, transtornos relacionados ao uso de } \\
\text { álcool, promoção da saúde }\end{array}$ \\
\hline 16 & 2017 & 4 & $\begin{array}{l}\text { ação intersetorial, drogas, prevenção primária, adolescente, saúde escolar, } \\
\text { avaliação de programas e projetos de saúde }\end{array}$ \\
\hline 17 & 2018 & 3 & adolescente em conflito com a lei, representação social, droga \\
\hline 18 & 2018 & 3 & $\begin{array}{l}\text { psicologia social, identidade, uso de drogas, pessoas em situação de rua, saúde } \\
\text { mental }\end{array}$ \\
\hline
\end{tabular}




\section{Motivos que levam ao consumo de álcool e outras drogas}

Bastante presente na revista Psicologia: Ciência $e$ Profissão é a busca dos motivos que levam ao consumo de substâncias psicoativas. Dos artigos em estudo, quatro têm esse foco de análise. Xavier (2005), por exemplo, analisa a associação entre religiosidade e consumo de álcool em uma população indígena, ressaltando a presença do uso em rituais religiosos e a existência de valores coletivos que incentivam o consumo de bebidas alcóolicas. Esse estudo aproxima-se do realizado por Maciel, Oliveira e Melo (2012), que analisa as representações sociais de profissionais de saúde quanto ao uso do álcool por índios Potiguara. Segundo as autoras, os profissionais associam o alcoolismo, p or um lado, a o processo de aculturação dos indígenas e, por outro, ao uso do álcool nas festividades e em rituais religiosos. Além disso, os profissionais c olocam o u so d o á lcool c omo porta de entrada para outros comportamentos de risco e atitudes violentas.

Já Conte, Oliveira, Henn e Wolff (2007) e studam a relação do consumismo com o uso de substâncias psicoativas e com a prática de atos ilícitos vinculados à venda e à distribuição de drogas. Destacam a forma como a criminalidade associa-se a outros processos de exclusão políticos e sociais, decorrentes da falta de acesso a educação de qualidade, de emprego e de renda. Por fim, os estudos de Medeiros, Pimentel, Monteiro, Gouveia e Medeiros (2015) têm como foco a base axiológica das atitudes frente ao uso de álcool, testando o modelo hierárquico valores $\rightarrow$ atitudes $\rightarrow$ comportamento. Os resultados indicam que valores de experimentação se correlacionam positivamente com atitudes favoráveis ao consumo de álcool, ao passo que valores normativos o fazem negativamente. Essas análises vão ao encontro dos demais estudos que compõem a subcategoria, no sentido de apontar para a presença de questões culturais, políticas e econômicas ligadas ao consumo individual e coletivo de drogas.

\section{Produção de verdades sobre os usuários de drogas}

Um segundo foco de estudos, presente em cinco dos 18 artigos analisados, é a produção de verdades sobre os usuários de drogas. Trata-se de pesquisas que estudam a construção de estigmas e preconceitos, seja dos próprios usuários sobre si, seja de profissionais de saúde ou da mídia sobre eles. Ao refletirem sobre os achados de suas pesquisas, as(es/os) autoras ${ }^{4}(\mathrm{es})$ destacam que essa produção não tem efeitos somente sobre os processos de subjetivação dos usuários, trabalhadores, gestores e sociedade em geral, uma vez que pauta o modo como nos relacionamos com as drogas e com as pessoas que delas fazem uso, além da construção de políticas e terapêuticas vinculadas ao tema. Por exemplo, a construção da ideia do usuário como sujeito passivo e sem controle frente às drogas, trazida em todos os artigos pesquisados, vai na contramão da lógica da redução de danos, reforçando o investimento em práticas de internamento coercitivas e o incremento de políticas proibicionistas e punitivistas. Ademais, incide sobre a descrença dos trabalhadores da saúde e da segurança em relação à possibilidade de obtenção de resultados positivos para os usuários.

Romanini e Roso (2012) analisam a ideologia subjacente à produção de uma campanha midiática sobre o crack e mostram a forma como os usuários da substância vão sendo descritos como sujeitos objetificados pela droga, passivizados por seu potencial avassalador e criminalizados em decorrência da dependência química. Evidenciam, ainda, a construção do crack como o inimigo social a ser enfrentado e reprimido por práticas proibicionistas. Já Silva e Peres (2014) analisam as concepções de profissionais de saúde de nível superior que atuam na Estratégia de Saúde da Família no que tange à atenção em saúde a usuários de álcool e outras drogas. De acordo com o estudo, a maioria dos profissionais assumiu uma postura moralista e preconceituosa em relação ao uso de drogas, apontando-o como a principal causa de uma ampla gama de malefícios para o indivíduo, a família e a sociedade. Os usuários são vistos pelos técnicos como pessoas que dificilmente demonstram adesão aos tratamentos e que deveriam ser passivas diante destes. Isso porque, para os profissionais, o conhecimento sobre as drogas e as terapêuticas está localizado nos trabalhadores da saúde, e não nos usuários.

\footnotetext{
${ }^{4}$ Referimo-nos às autoras prioritariamente no feminino, não somente por corroborar a feminização da linguagem assumida pelo Sistema Conselhos de Psicologia, mas também porque, nesta pesquisa em específico, das 46 pessoas autoras dos 18 artigos em análise, 31 identificam-se por nomes reconhecidos como femininos, enquanto 15 fazem uso de nomes culturalmente associados ao masculino. De toda forma, optamos por manter a presença não só do masculino, como também do gênero neutro dentro dos parênteses, para marcar a presença de pessoas não binárias na construção da Psicologia como ciência e profissão.
} 
Os outros três artigos inseridos nesta categoria tratam da percepção de pessoas que fazem uso de drogas a respeito das drogas e seus usuários. Melo e Maciel (2016) buscaram conhecer as representações sociais de usuários de drogas sobre essas pessoas. Segundo as autoras, os usuários são concebidos como sujeitos não confiáveis, maus-caracteres, doentes sem capacidade de lutar contra sua dependência. Rodrigues, Lima e Holanda (2018) discutem o modo como pessoas que vivem em situação de rua e que fazem uso abusivo de drogas lidam com os processos de exclusão social aos quais estão submetidas, observando de que forma os personagens que representam repercutem no reconhecimento de suas identidades. Os autores denunciam a negação dos direitos a participação social, da autonomia e de possibilidades de essas pessoas se reconhecerem em outros personagens que não apenas os de usuários de drogas. Por fim, Andrade, Alves e Bassani (2018) analisaram as representações sociais do uso de drogas entre os adolescentes em conflito com a lei e a existência da relação com o ato infracional. A droga foi representada pelos adolescentes como responsável por trazer consequências relacionadas a problemas de saúde e conflitos familiares. O crack foi citado como a droga mais destruidora e vinculada aos atos delitivos para manutenção do vício.

\section{Prevenção e tratamento}

Esta foi a subcategoria com maior volume de artigos, composta por seis dos 18 estudos analisados. Lopes, Peuker e Bizarro (2013) analisaram a aplicação de um programa de cessação do tabagismo realizado em uma empresa de transporte coletivo urbano. $\mathrm{O}$ artigo apresenta um modelo de intervenção de abordagem cognitivo-comportamental passível de ser implantado no contexto organizacional. Ferreira e Sartes (2015), por sua vez, fazem uma revisão sistemática de artigos científicos sobre intervenções realizadas no ambiente de trabalho para o uso de drogas. Concluem pela insuficiência de evidências científicas que validem as intervenções apresentadas e sua aplicabilidade no contexto de trabalho. Ambos os artigos, ao sustentarem a importância do desenvolvimento de terapêuticas para o uso de drogas no ambiente de trabalho, fazem-no em nome da melhoria da produtividade e da diminuição do absenteísmo. Nesse mesmo sentido, diversos dos artigos analisados, ao justificarem sua importância, trazem dados sobre os custos que os tratamentos de pessoas que fazem uso de drogas geram ao país. Isso evidencia a maneira como as terapêuticas em torno do uso de drogas não são pensadas somente como problemas técnicos no âmbito da saúde, mas como problemas econômicos para as empresas e o próprio Estado.

Outro ponto interessante, presente tanto nos artigos referidos acima quanto nos estudos de Almeida e Roazzi (2014), de Scaduto, Barbieri e Santos (2015) e de Reis, Machado, Andrade e Figueiredo (2017), diz respeito ao desenvolvimento de pesquisas que buscam mostrar a efetividade das técnicas e terapêuticas empregadas nos mais diversos ambientes para prevenção e tratamento do uso abusivo de drogas. Almeida e Roazzi (2014), por exemplo, investem na produção de dados que respaldam o uso da Teoria da Ação Racional e de diferentes formas de comunicação não persuasiva para a predição da intenção de adotar o comportamento de não uso de bebidas alcoólicas e direção em estudantes universitários. Scaduto et al. (2015) estudam a vinculação de internos aos princípios de uma comunidade terapêutica (CT) e sua relação com o processo de mudança vivenciado ao longo do tratamento. Os resultados do estudo indicam melhor vínculo dos participantes com os princípios da CT, em geral, ao final da internação. Já Reis et al. (2017) buscam avaliar a efetividade de um curso de atualização para os profissionais que trabalham na Atenção Primária em Saúde (APS), tendo em vista o conhecimento e as formas de abordagem frente ao uso problemático do álcool, ressaltando a importância de atualização dos profissionais.

Ainda no âmbito dos serviços de atenção às pessoas que fazem uso de drogas, Peres, Grigolo e Schneider (2017) estudam os desafios da articulação intersetorial para a implantação de um programa de prevenção ao uso de drogas. Para as autoras, os profissionais estudados perceberam que as ações conjuntas entre saúde e educação possibilitaram ampliar o cuidado às famílias que procuravam a UBS e facilitaram o acesso integral aos educandos. No entanto, o atravessamento de questões políticas, a personalização das relações intersetoriais, as dificuldades de compreender o significado da intersetorialidade na prática cotidiana dos serviços e as excessivas demandas cotidianas de trabalho que impedem o envolvimento dos profissionais em novos projetos ainda são apontados como desafios ao trabalho em rede. 


\section{Análise das políticas sobre drogas}

A última subcategoria é composta por três artigos, tendo como foco a análise de políticas que integram o campo das políticas sobre drogas. Importante destacar que todos os 18 artigos fazem referência a um ou mais dos marcos legais que orientam as políticas sobre drogas e, em sua maioria, entendem que suas análises podem contribuir para a construção e a qualificação de ações estatais, ainda que esse não seja o objetivo central dos referidos artigos.

No âmbito das políticas sobre drogas, Cheibub (2006) volta-se para o campo da segurança pública e do sistema prisional, evidenciando como o uso de drogas vai se constituir como uma ferramenta de promoção de práticas disciplinares de classificação dos sujeitos e de produção da norma e do normal dentro e fora das prisões. Ainda no âmbito das políticas de segurança, Nascimento e Menandro (2016) buscaram conhecer a percepção de policiais militares sobre um programa de fiscalização de trânsito implementado antes mesmo da Lei Seca. Os policiais afirmaram haver valorização do beber excessivo e menosprezo ao risco existente na associação de bebida alcóolica com a direção, além de trazerem informações sobre dificuldades e realizações das ações educativas e fiscalizatórias.

Machado e Boarini (2013), por sua vez, realizaram um resgate histórico da estratégia de redução de danos (RD) e de sua presença nas políticas sobre drogas no Brasil. Em seu estudo, apontam que o descompasso histórico entre as políticas sobre drogas oriundas do campo da segurança e aquelas provindas da saúde pública, com predominância das de cunho proibicionista, bem como o preconceito vigente em relação ao usuário de drogas, constituem entraves ao avanço das práticas de redução de danos. Tal discussão dialoga com as produções trazidas na primeira subcategoria, sobre os estigmas e preconceitos que se inscrevem nas pessoas que fazem uso de drogas.

\section{Compromissos da Psicologia com a produção de saberes sobre drogas}

A análise dos artigos publicados nos últimos 14 anos na revista Psicologia: Ciência e Profissão auxilia-nos a dar visibilidade à forma como esse campo do saber tem sido chamado a se inserir na produção de verdades sobre as drogas, ligando-se prioritariamente a quatro questões: a identificação das causas do uso; a compreensão da construção de representações e estigmas em torno dos usuários; a produção e a legitimação de terapêuticas a serem empregadas na prevenção e no tratamento; e a elaboração de políticas públicas, com destaque às áreas da saúde, do trabalho, da educação, da assistência social e da segurança. A grande maioria dos artigos estudados coloca-se no sentido de responder a essa demanda. Ao fazerem isso, essas produções atuam reforçando o lugar das drogas como elemento central e fundamental de discussão, reconhecido efetivamente como o problema a ser enfrentado.

Interessante destacar que em quase todas as publicações há a afirmação de que a relação problemática com o uso está atrelada a questões políticas, econômicas e culturais, tais como: o uso em festividades e em práticas religiosas; o consumismo; os valores positivos associados ao uso; e os diversos processos de exclusão e de vulnerabilização sociais. No entanto, dentro do campo de produção de intervenções, as drogas seguem mantidas no lugar de visibilidade como o foco do problema, e não como um sintoma das questões anteriormente citadas.

O uso de drogas sempre fez parte da experiência humana, estando presente em todas as culturas, associado a práticas recreativas, lúdicas, religiosas e terapêuticas, constituindo-se como uma das formas de lidar com o prazer e com o sofrimento (Escohotado, 2009). Contemporaneamente, as drogas passaram a ser tomadas como um grande problema social, representado como um perigo que ameaça toda a sociedade. Ao mantermos as drogas nesse lugar e foco de nosso olhar, deixamos de colocar em questão os desinvestimentos nas políticas socioassistenciais, deixamos de pautar o desmantelamento da rede de atenção e dos serviços já existentes no âmbito do Sistema Único de Saúde.

Alinhadas a isso, as políticas sobre drogas caminham em direção ao encolhimento do investimento na rede pública para a destinação de recursos a equipamentos privados, tais como as comunidades terapêuticas. Ademais, nas recentes transformações das políticas sobre drogas, vemos o acirramento de ações que se inscrevem exclusivamente nos indivíduos, como se o uso problemático fosse decorrente unicamente da associação entre o indivíduo e a droga e não estivesse atrelado a questões multifacetadas, ou como se estas últimas fossem questões secundárias e menos importantes do que a desintoxicação ou o afastamento físico de usuários das cenas de uso. 
Em 1998, a Assembleia da ONU previu "um mundo livre de drogas" em dez anos a partir do investimento em políticas repressivas de guerra às drogas. Em 2008, observou-se o aumento do comércio e do consumo de drogas tornadas ilícitas (CFP \& Crepop, 2019). Essas ações geraram gastos bilionários aos Estados envolvidos e tiveram como consequência, ainda, o aumento da violência letal (Oliveira \& Ribeiro, 2018). No Brasil, além de esse aumento concentrar-se na população jovem, pobre e negra, viu-se também como efeito das políticas proibicionistas o encarceramento em massa, em especial desta população, demonstrando a face racista e genocida da guerra às drogas.

Frente a isso, destaca-se aqui a importância de não respondermos de forma tecnicista às questões que nos são colocadas, mas de nos implicarmos no necessário exercício de reflexão sobre o modo como formulamos os problemas a serem enfrentados e sobre os jogos de interesse, poder e verdade dos quais essa dada formulação está a serviço. Ao invertermos as perguntas que nos são impostas e tomarmos, por exemplo, os processos de exclusão e vulnerabilização sociais como problemas a serem pautados pelos saberes psis, pelas campanhas midiáticas ou pelas ações estatais, talvez tenhamos uma incidência maior no enfrentamento ao uso problemático e à criminalização de adolescentes e de pessoas em situação de rua.

Nesse sentido, reforça-se a importância de investirmos na produção de conhecimentos situados ética e politicamente a serviço do cuidado em liberdade e na defesa de um sistema público de saúde. Finalmente, gostaríamos de destacar que, uma vez que não acreditamos na neutralidade do conhecimento científico, mas reafirmamos que este possui efeitos políticos e que a reflexão sobre os efeitos daquilo que produzimos é um exercício fundamental à Psicologia, resta o desafio à revista Psicologia: Ciência e Profissão de investir na construção de um escopo que acolha e impulsione a diversidade de saberes psicológicos, sem perder de vista o próprio compromisso que o Sistema Conselhos de Psicologia tem assumido ao longo dos anos - o de defender uma profissão implicada com a garantia de direitos, com o fortalecimento das políticas públicas e com a promoção de cuidado em liberdade.

\section{Referências}

Almeida, N. D., \& Roazzi, Antonio. (2014). Álcool e direção em universitários, comunicação persuasiva e prevenção. Psicologia: Ciência e Profissão, 34(3), 715-732. https://doi.org/10.1590/1982-3703000632013

Andrade, S. F. O., Alves, R. S. F., \& Bassani, M. H. P. A. (2018). Representações sociais sobre as drogas: um estudo com adolescentes em conflito com a lei. Psicologia: Ciência e Profissão, 38(3), 437-449. https://doi.org/10.1590/1982-37030000742017

Cheibub, W. B. (2006). Práticas disciplinares e usos de drogas: a gestão dos ilegalismos na cena contemporânea. Psicologia: Ciência e Profissão, 26(4), 548-557. http://pepsic.bvsalud.org/scielo.php?script=sci_arttext\&pid= S1414-98932006000400003\&lng=pt\&tlng=pt

Conselho Federal de Psicologia. (2010). Subjetividade do consumo de álcool e outras drogas e as políticas públicas brasileiras. Brasília: CFP. https://site.cfp.org.br/wp-content/uploads/2010/06/AlcoolDrogas_novas_alteracoes.pdf

Conselho Federal de Psicologia. (2011). Relatório da $4^{a}$ Inspeção Nacional de Direitos Humanos: locais de internação para usuários de drogas. Brasília: CFP. https://site.cfp.org.br/wp-content/uploads/2012/03/2a Edixo_relatorio_inspecao_VERSxO_FINAL.pdf

Conselho Federal de Psicologia. (2012). Drogas e cidadania:em debate. Brasília: CFP. https://site.cfp.org.br/wp-content/uploads/2013/02/Drogas-e-cidadania.pdf

Conselho Federal de Psicologia. (2014). Posicionamento político do Conselho Federal de Psicologia relativo à política de drogas. Brasília: CFP. https://site.cfp.org.br/wp-content/uploads/2014/12/Posicionamentopol\%C3\%ADtico-do-CFP-relativo-à-Pol\%C3\%ADtica-de-Drogas.pdf

Conselho Federal de Psicologia. (2018). Os retrocessos da política de drogas no Brasil: nota do Sistema Conselhos de Psicologia sobre recentes ações do Conad. https:/ / site.cfp.org.br/os-retrocessos-da-politica-de-drogas-no-brasil/

Conselho Federal de Psicologia \& Centro de Referências Técnicas em Psicologia e Políticas Públicas. (2019). Referências técnicas para atuação de psicólogas(os) em políticas públicas de álcool e outras drogas. Brasília, DF: CFP; Crepop. https://site.cfp.org.br/wp-content/uploads/2019/09/AlcooleOutrasDrogas_web-FINAL.pdf 
Conselho Federal de Psicologia, Mecanismo Nacional de Prevenção e Combate à Tortura, \& Procuradoria Federal dos Direitos do Cidadão/Ministério Público Federal. (2018). Relatório da Inspeção Nacional em Comunidades Terapêuticas: 2017. Brasília, DF: CFP; MNPCT; MPF. https://site.cfp.org.br/wp-content/uploads/2018/06/ Relatorio-da-inspecao-nacional-em-comunidades-terapeuticas_web.pdf

Conte, M., Oliveira, C. S., Henn, R. C., \&Wolff, M. P. (2007). Consumismo, uso de drogas e criminalidade: riscos e responsabilidades. Psicologia: Ciência e Profissão, 27(1), 94-105. https://doi.org/10.1590/S1414-98932007000100008

Escohotado, A. (2009). Historia elemental de las drogas. Barcelona: Anagrama.

Ferreira, M. L. \& Sartes, L. M. A. (2015). Intervenções realizadas no ambiente de trabalho para o uso de drogas: revisão sistemática. Psicologia: Ciência e Profissão, 35(1), 96-110. https://doi.org/10.1590/1982-3703001802013

Guareschi, Galeano, \& Bicalho (2020). 40 anos: O que a Psicologia tem produzido enquanto ciência e profissão?. Psicologia: Ciência e Profissão, 40, 1-12. https://doi.org/10.1590/1982-3703003237742

Lei $n^{\circ}$ 8.069, de 13 de julho de 1990. (1990). Dispõe sobre o Estatuto da Criança e do Adolescente e dá outras providências. Brasília, DF: Presidência da República.

Lei $n^{\circ}$ 11.343, de 23 de agosto de 2006. (2006). Institui o Sistema Nacional de Políticas Públicas sobre Drogas - Sisnad; prescreve medidas para prevenção do uso indevido, atenção e reinserção social de usuários e dependentes de drogas; estabelece normas para repressão à produção não autorizada e ao tráfico ilícito de drogas; define crimes e dá outras providências. Brasília, DF: Presidência da República.

Lei $n^{\circ} 13.840$, de 5 de junho de 2019. (2019). Altera as Leis no 11.343, de 23 de agosto de 2006, 7.560, de 19 de dezembro de 1986, 9.250, de 26 de dezembro de 1995, 9.532, de 10 de dezembro de 1997, 8.981, de 20 de janeiro de 1995, 8.315, de 23 de dezembro de 1991, 8.706, de 14 de setembro de 1993, 8.069, de 13 de julho de 1990, 9.394, de 20 de dezembro de 1996, e 9.503, de 23 de setembro de 1997, os Decretos-Lei no 4.048, de 22 de janeiro de 1942, 8.621, de 10 de janeiro de 1946, e 5.452, de $1^{\circ}$ de maio de 1943, para dispor sobre o Sistema Nacional de Políticas Públicas sobre Drogas e as condições de atenção aos usuários ou dependentes de drogas e para tratar do financiamento das políticas sobre drogas. Brasília, DF: Presidência da República.

Lopes, F. M., Peuker, A. C., \& Bizarro, L. (2013). Aplicação de um programa de cessação do tabagismo com rodoviários urbanos. Psicologia: Ciência e Profissão, 33(2), 490-499. https://doi.org/10.1590/S1414-98932013000200017

Machado, L. V. \& Boarini, M. L. (2013). Políticas sobre drogas no Brasil: a estratégia de redução de danos. Psicologia: Ciência e Profissão, 33(3), 580-595. https://doi.org/10.1590/S1414-98932013000300006

Maciel, S. C., Oliveira, R. C. C., \& Melo, J. R. F. (2012). Alcoolismo em indígenas potiguara: representações sociais dos profissionais de saúde. Psicologia: Ciência e Profissão, 32(1), 98-111. https://doi.org/10.1590/ S1414-98932012000100008

Medeiros, E. D., Pimentel, C. E., Monteiro, R. P., Gouveia, V. V., \& Medeiros, P. C. B. (2015). Valores, atitudes e uso de bebidas alcoólicas: proposta de um modelo hierárquico. Psicologia: Ciência e Profissão, 35(3), 841-854. https://doi.org/10.1590/1982-3703001532013

Melo, J. R. F. \& Maciel, S. C. (2016). Representação social do usuário de drogas na perspectiva de dependentes químicos. Psicologia: Ciência e Profissão, 36(1), 76-87. https://doi.org/10.1590/1982-3703000882014

Nascimento, A. S. \& Menandro, P. R. M. (2016). Bebida alcoólica e direção automotiva: relatos de policiais militares sobre a “Lei Seca”. Psicologia: Ciência e Profissão, 36(2), 411-425. https://doi.org/10.1590/1982-370300067201

Oliveira, N. \& Ribeiro, E. (2018). O massacre negro brasileiro na guerra às drogas. SUR 28-Revista Internacional de Direitos Humanos, 15(28), 35-43. https://sur.conectas.org/wp-content/uploads/2019/05/sur-28-portugues-nathalia-oliveira-e-eduardo-ribeiro.pdf

Peres, G. M., Grigolo, T. M., \& Schneider, D. R. (2017). Desafios da intersetorialidade na implementação de Programa de Prevenção ao Uso Abusivo de Drogas. Psicologia: Ciência e Profissão, 37(4), 869-882. https://doi.org/ $10.1590 / 1982-3703003272016$

Portaria MS no 3.088, de 23 de dezembro de 2011. (2011). Institui a Rede de Atenção Psicossocial para pessoas com sofrimento ou transtorno mental e com necessidades decorrentes do uso de crack, álcool e outras drogas, no âmbito do Sistema Único de Saúde (SUS). Brasília, DF: Ministério da Saúde.

Reis, F. G., Machado, E. C., Andrade, M., \& Figueiredo, G. L. A. (2017). Conhecimento e abordagens acerca do uso problemático do álcool. Psicologia: Ciência e Profissão, 37(2), 335-348. https://doi.org/10.1590/1982-3703000852016 
Rodrigues, J. S., Lima, A. F., \& Holanda, R. B. (2018). Identidade, drogas e saúde mental: narrativas de pessoas em situação de rua. Psicologia: Ciência e Profissão, 38(3), 424-436. https://doi.org/10.1590/1982-37030004912017

Romanini, M., \& Roso, A. (2012). Mídia e crack: promovendo saúde ou reforçando relações de dominação?. Psicologia: Ciência e Profissão, 32(1), 82-97. https://doi.org/10.1590/S1414-98932012000100007

Scaduto, A.A., Barbieri,V., \&Santos, M.A. (2015).Adesãoaos princípios da comunidadeterapêuticaeprocessodemudança ao longo do tratamento. Psicologia: Ciência e Profissão, 35(3), 781-796. https://doi.org/10.1590/1982-3703000792013

Silva, W. R., \& Peres, R. S. (2014). Concepções sobre álcool e outras drogas na atenção básica: o pacto denegativo dos profissionais de saúde. Psicologia:CiênciaeProfissão, 34(2), 474-487.https://doi.org/10.1590/1982-3703000492013

Xavier, M. (2005). Religiosidade e problemas com o álcool: um estudo de caso. Psicologia: ciência e profissão, 25(1), 88-99. http://pepsic.bvsalud.org/scielo.php?script=sci_arttext\&pid=S1414-98932005000100008\&lng=pt\&tlng=pt

\section{Carolina dos Reis}

Professora do Programa de Pós-Graduação em Psicologia Social e Institucional da Universidade Federal do Rio Grande do Sul, onde integra do Núcleo de Estudos em Políticas e Tecnologias Contemporâneas de Subjetivação Núcleo E-politics. Membro da Comissão Editorial da revista Psicologia: Ciência e Profissão (2018-2020). Conselheira do Conselho Regional de Psicologia do Rio Grande do Sul, Gestão Frente em Defesa da Psicologia (2019-2022). Porto Alegre - RS. Brasil.

E-mail: carolinadosreis@gmail.com

(1) https://orcid.org/0000-0001-6482-2677

Endereço para envio de correspondência:

Rua Doutor Alcides Cruz, 100/401, Santa Cecília. CEP 90630-160, Porto Alegre - RS, Brasil.

Recebido 07/05/2020

Aceito 16/06/2020

Received 05/07/2020

Approved 06/16/2020

Recibido 07/05/2020

Aceptado 16/06/2020

Como citar: Reis, C. (2020). Implicações da Produção do Conhecimento em Psicologia sobre Álcool e Outras Drogas. Psicologia: Ciência e Profissão, 40, 1-10. https://doi.org/10.1590/1982-3703003237729

How to cite: Reis, C. (2020). Implications of the Production of Psychological Knowledge on Alcohol and Other Drugs. Psicologia: Ciência e Profissão, 40, 1-10. https://doi.org/10.1590/1982-3703003237729

Cómo citar: Reis, C. (2020). Implicaciones de la Producción de Conocimiento en Psicología sobre el Alcohol y Otras Drogas.. Psicologia: Ciência e Profissão, 40, 1-10. https://doi.org/10.1590/1982-3703003237729 